\title{
An Autonomous UAS with AI for Forest Fire Prevention, Detection, and Real Time Advice and Communication To and Among Firefighters
}

\author{
Yfantis $\mathbf{E A}^{1^{*}}$ and Harris $\mathrm{SL}^{2}$ \\ ${ }^{1}$ Computer Science Department, University of Nevada, Las Vegas, USA \\ ${ }^{2}$ Department of Computer and Electrical Engineering, University of Nevada, Las Vegas, USA
}

Received: September 16, 2017; Accepted: September 25, 2017; Published: October 05, 2017

*Corresponding author: Yfantis EA, Professor, Computer Science Department, University of Nevada, Las Vegas, USA, E-mail: yfantis@cs.unlv.edu

\begin{abstract}
Forest fires are increasing annually due to global warming. The cause of most forest fires are humans, followed by lightning, and winds enabling dry debris, such as small pieces of dry wood that rub against each other creating a spark. Forest fires are catastrophic events that annually claim lives, destroy property, kill domestic and wild animals, and destroy the trees and vegetation that provide oxygen, filter the water, food for animals, and playgrounds and shelter for humans and animals. Additionally after fires burn a forest, rain water runs down the mountain forming mudslides that often times cause damages to land, houses, other building that are on their way, cars, and other property and also kill people, and animals. Therefore forest fires are a disaster having negative effects on people, animals and flora in many ways. In this research paper we provide a solution to preventing forest fires by monitoring the forest using an autonomous UAS powered by solar panels, batteries and a brushless motor, equipped with two high quality cameras in the visual range an infrared camera and a LIDAR, capable of recognizing dry brash, people making camp fires, dead trees, and providing that and other relevant information via wireless transmission to the personnel designated to protect the forest. The intelligent system of the UAS is embedded into a Stratix-10 IntelAltera FPGA that is capable of performing 29.5 teraflops sustained, and is capable of detecting forest fires, as well as providing real time advice to the fire fighters how to efficiently fight the fire. In addition to that the UAS has a router a transceiver and an antenna, enabling the firefighters to communicate with each other and with command and control when the fire destroys the ground antenna system.
\end{abstract}

Keywords: AI; Neural networks; Signal processing; Sensor networks;

\section{Introduction}

In this research paper we present a solution to the forest fire problem. Our solution includes fire prevention, early fire detection, and an artificial intelligent system having perception, learning ability, reasoning, and ability to communicate. The ability to communicate replaces the natural language requirement of the classical artificial intelligent systems. Our system includes autonomous unmanned air system UAS with a $8 \mathrm{ft}$ wing span and $8.31 \mathrm{ft}$ fuselage, powered by solar energy recharging a lithium ion battery system which powers the 1.5 horse power (hp) electric motor enabling the propeller airplane to carry 30 pounds gross weight including the electronics needed for the airplane to fly, and its instrumentation. The instrumentation of the airplane includes two smart high quality smart cameras in the visual range having the ability to pan tilt and zoom (ptz) connected to a stratix-10 Intel-Altera FPGA programed by our team to provide the camera intelligence. The airplane communicates with our Wireless Sensor Network in the ground (WSN) and gets climatological information such as temperature, humidity, wind direction and speed, rain, $\mathrm{CO}, \mathrm{CO}_{2}$, levels, areas with dry brush and dead that could be fuel to the fire. One Part of prevention is taking all these parameters into account and computing the probability of forest fire given these conditions. The metrics we develop is an alternative to Fire Weather Index (FWI) developed by the Canadian forest service. One of the three fuel classes the FWI estimate as a function of the moisture, is the Fine Fuel Moisture Code (FFMC), which is used as an indicator to ease of ignition. Considering that most of the forest fires are caused by humans, some intentional and some accidental due to negligence, the other part of prevention is to monitor the forest in order to detect humans making camp fires or other type of fires. In either case inform the forest personnel responsible for enforcing the laws and for taking care of the forest to act in order to avoid forest fires. The system has several wireless communication systems all based on the IEEE 801.11, one communication system is between the airplane and the Wireless Sensor Network (WSN) in the ground another between the airplane and the firefighters, also a third one between the airplane and the forest care takers, and a fourth one that enables communication between the firefighters in case of destruction of their communication infrastructure. Fire detection is performed at the camera level. The two cameras in the visual range as well as the infrared camera, and the WSN data are input to a stratix-10 Intel FPGA which during the day time performs computational photography creating an accurate 3-D representation of the field of view, does digital image processing, pattern recognition cross-correlating the visual data with the SWN data from the ground and recognizes if there are any fires in the forest pinpointing their location very accurately. It also transmits the message to the emergency response team and the 
firefighters in the area. During the night ours the near infrared band of the visual range cameras is captured only, their output is cross correlated with that of the infrared camera and that of the WSN, by the stratix-10 FPGA and the information in case of fire is transmitted to the first responders and the firefighters. In case of a forest fire the airplane has the intelligence to follow the fire, create an intelligent plan of action, and transmit that to the firefighters giving them advice about fighting the fire, interrogating the transponders of other aircraft so that it will stay away from their path and help establish communication among the firefighters and command and control. This paper is structured as follows. First the abstract, followed by the introduction, followed by the background information, followed by the UAV with the communications and AI systems, followed by conclusions, and finally the reference.

\section{Background Information}

Climate change, along with the population increase which increases the probability of forest fires due to human causes, have been producing more fires in the recent years than the previous years. The state of Nevada and many other western states had many catastrophic fires destroying many hectares (ha) of forest. Technology has made much advancement in the recent years that if we were to apply them in prevention, detection, and reduction of the catastrophic effect of forest fires could reduce the catastrophic effects of forest fires by a large percentage. There has been a great deal of research in this area that if they were to put into play could alleviate the forest fire situation. One of the earliest researches to detect fires is due to Cappellini, et al. [1]. This was based digital image thresholding. The probability of misclassification of this system is high. Since then many other research have been published in the area of fire detection recommends conventional cameras installed on towers to provide a continuous sequence of video frames of an area triggering an alert in case of fire [2]. The systems consist of the imagers, a computer for image processing, the software, and a communication network to transmit the information. Over the years many research in the area of forest fire have been published [3-6]. Recent research in this area focus on RoTs which are flames and smoke based on the calculation of color indices, In their work the images obtained in the RGB space are transformed into grey scale, a thresholding process is used separating the region of interest from the rest of the image [7]. In addition to that two indices have been developed the Fire Detection Index (FDI) and the Forest Fire Detection Index (FFDI). The author's first compute the Vegetation Index (VI) used to classify different plants. Also they compute the Excess Green colors ExG $=2 \mathrm{~g}-\mathrm{r}-\mathrm{b}$. There has also been a great deal of research related to forest fire detection systems based on wireless sensor networks. Some of the WSNs use cameras, fire detection sensors and the wireless communication protocol 802.11g [8]. In order to provide a metric relating to the potential for wild fire the Canadian fire weather index is often used [10].

There has been research related to using UAVs or UASs [9], most of them focus on fire detection and tracking. NASA in collaboration with USDA Forest Service [11], proposed the use of UAV technology in forest service resource management.
Forest service chief Dale Bosworth identified four threats to our nation's forests: i) fire and fuels, ii) invasive species, iii) loss of open space, iv) unmanaged recreation. The forest service aviation own and operates 44 aircraft and helicopters, contracts with over 800 aircraft and helicopters annually, supports forest service and other land management agencies missions including: i) forest health, ii) law enforcement, iii) resource management, vi) fire surveillance, v) Aerial reconnaissance vi) Delivery of smokejumpers, vii) Firefighter and cargo transport vii) Aerial delivery of retardant and water [11]. There has been a plethora of research papers related to WSN and some related to remote sensing; the analytics on most of these papers are based mainly on digital image processing [11]. Our work uses digital image processing, and signal processing in general, as the first step, followed by pattern recognition, neural networks, and genetic algorithms to create a complex and efficient deep learning solution to the problem.

\section{An Autonomous UAV with AI for Fire Prevention, Fire Detection, Real Time Adviser to the Firefighters, and for Enabling Real Time Communication}

\section{Protect the forests, and the instrumentation on the UAV}

Forests purify the air we breathe, purify the water, recycle nutrients, stabilize the soil, create habitat for wildlife, provide recreation for humans, and nurture environments rich in biological diversity. Unfortunately many millions of forest hectares are being destroyed by fires each year. To help reduce the catastrophic effects of forest fires we present a solution using solar powered UAVs instrumented with two visual range cameras each with a high quality imager chip having a near infrared channel, an infrared camera, the three of which are connected to a stratix-10 Intel-Altera FPGA for real time image processing and classification. We use the SODAR systems to measure the wind speed at various heights above the ground. The purpose of our technology is to improve forest fire imaging and mapping, is capable of detecting very small heat sources over large areas, capable of mapping large fires very accurately, capable of performing large area mapping, uses multispectral analysis, provides tactical and real time decisions operates on fixed wing aircraft. The acquisition and operating cost of our UAV system is is far less to the current technology. It has autonomous flight capabilities, long flight duration (long endurance), real-time transmission of images and video. Capable of night operations, flight can be tracked real time with antennas, or portable tracking radar. We also have Sense and Avoid technology to satisfy FAA safety requirements. Includes sensors, data telemetry, information processing and intelligent mission management to acquire pertinent scientific and disaster management data over wildfires spread throughout the western US during a 24-hour period. Vector to loiter over fires and real-time flight profiling, on board real-time image geo-rectification and processing, and real time telemetry. The ground WSN consists of robust inexpensive sensors with flash memory, and a transceiver to transmit data 
using our modified wireless transmission 801.11 protocols for wireless communication at $925 \mathrm{MGH}$ license-exempt frequencies ISM band, powered by vibrating crystals, solar panels and rechargeable batteries [12]. Some sensors sense the temperature, others the wind direction and speed, others the humidity, others the carbon monoxide, and carbon dioxide levels, Each sensor has two batteries, a small battery to keep the device that accepts signals from the airplane and a larger battery to keep the whole device on and operational. When the sensor gets the airplane signal the small battery wakes up the device and transmits its information to the airplane, along with its GPS coordinates.

\section{The Airplane}

The airplane design. The main reason we chose an airplane versus a multicopter is because the airplane can fly much longer than a multicopter using less power than a multicopter, and is more stable in rough weather than a multicopter. The main reason for designing and building our own airplane are the following:

-No existing design does what we want our airplane to do.

-Designing and building it once makes it to modify it and repair it.

-We have the theoretical knowledge and some experience in designing, building, and flying unmanned airplanes and multicopters.

-Having the knowledge and experience of designing and building the airplane it is easy to improve the design and build a better airplane.

-It is much cheaper to build one ourselves than outsourcing the design and building.

Design requirements:

-Stable but responsive.

-Performance at least as good as other unmanned aircraft.

-Solar powered, with battery backup to keep on flying for days.

-Capable of carrying the instruments and its own weight.

-Electric motor.

-One propeller

-Parachute, in case of engine failure or an accident.

\begin{tabular}{|c|c|c|}
\hline & Goal & Threshold \\
\hline $\begin{array}{l}\text { Max Load (Butteries } \\
\text { +Instrumentation) }\end{array}$ & $30 \mathrm{lbs}$ & $20 \mathrm{lbs}$ \\
\hline Maximum Speed & 50 kts (knots) & $45 \mathrm{kts}$ \\
\hline Range & Fly forever & 80kts (on battery only) \\
\hline Maximum Cruise Speed & 50 kts (nautical miles) & $40 \mathrm{kts}$ \\
\hline Rate of Climb-Sea Level & $100 \mathrm{ft} / \mathrm{sec}$ & $80 \mathrm{ft} / \mathrm{sec}$ \\
\hline Stall Speed-flaps down & $30 \mathrm{kts}$ & $30 \mathrm{kts}$ \\
\hline Minimum Control Speed & 30 kts & $30 \mathrm{kts}$ \\
\hline Takeoff Distance & $25 \mathrm{ft}$ & $30 \mathrm{ft}$ \\
\hline Landing Distance & $25 \mathrm{ft}$ & $30 \mathrm{ft}$ \\
\hline Cruise Speed & $45 \mathrm{kts}$ & $40 \mathrm{kts}$ \\
\hline Signal Transmission Distance & 50 Miles & 40 Miles \\
\hline Retract-Gear Smooth Design & $\frac{W}{h p}=680 * V_{\max }^{-0.79}$ & \\
\hline
\end{tabular}

Design and implementation method the dynamic pressure is $q=\frac{1}{2} \rho V^{2}(1)$ and is directly related to the lift. In this equation $\rho$ is the air density at the current flying altitude. The power loading to meet our goal of $455 \mathrm{kts}$ maximum speed is

$$
\frac{W}{h p}=680 *(50)^{-0.79}=30.93 \frac{l b s}{\text { horsepower }}
$$

Let $\mathrm{S}$ be the wing area, $\mathrm{L}$ be the lift the the lift coefficient $C_{L}=\frac{L}{q S}$ In order for the airplane to fly the lift $\mathrm{L}=\mathrm{W}$ (4). From equations (3) and (4) we have $\frac{W}{S}=q^{*} C_{L \max }=\frac{1}{2} \rho V_{\text {Stall }}^{2} C_{\text {Lmax }}(5) \mathrm{W} / \mathrm{S}$ is the wing loading. For our design stall speed is $10 \mathrm{kts}$, takeoff air density $=0.00238 \frac{\text { slugs }}{f t^{3}}, C_{\text {Lmax }}=1.6$ then from formula (1) the dynamic preasure is $3.055 \mathrm{psf}$ and the wing loading is $\frac{W}{S}=4.89 \mathrm{psf}$. The airplane weight $W_{0}=W_{e}+W_{\text {Max-Load }}=8+30=38$ lbs (6) where

$W_{0}$ is the total weight, $W_{e}$ is the weight of the airplane with the electric motor, propeller, solar panels, and the electronics needed for flying, and $W_{\text {Max-Load }}$ is the load of the instruments with their (3) batteries. The horse power of the motor needed is

$$
H p=\frac{W_{0}}{\text { Power Loading }}=\frac{38}{30.93}=1.229
$$


The wing area

$$
S=\frac{W_{0}}{\text { Wing Loading }}=\frac{38}{4.89}=7.77347 \mathrm{ft}^{2}
$$

So the wing span will be $8 \mathrm{ft}$, and the wing width $1 \mathrm{ft}$. It will be a high wing design to provide better protection to the instruments during takeoff and landing. This is a preliminary design. The final design will be very close to this providing that the specifications do not change. The final design will include the size and the number of blades of the propeller, the size of the horizontal and vertical stabilizers, the size of the ruder, the size of the elevator, the size of the flops, and the size of the ailerons.

The fuselage length is

$$
F L=3.6^{*} W_{0}^{0.23}=38^{0.23}=8.31 f t
$$

The electronics needed to fly the airplane are: $1.5 \mathrm{hp}$ electrical brushless motor, connected to a lithium-ion battery, via an electronic speed controller. Stepper motors controlled connected to the flops, aileron, rudder, and elevator, controlled by an AVR microcontroller, taking instructions from the ground station via the transceiver antenna of the airplane. The uninstrumented airplane will also have GPS, and a gyro.

\section{The Communications System}

The wireless communication protocol used is the ISM (Industrial Scientific Medical) with a modified forward error correction and automatic repeat request. The communication frequency between WSN and the airplane is $925 \mathrm{MHz}$, while all other communications frequencies vary between $5.5-10 \mathrm{GHz}$, using digital modulation and frequency hopping. The protocol modification is in the variable packet size optimization to increase the throughput, packet receive acknowledgement, and automatic repeat request. Wireless transmission is inherently noisy, especially in the presence catastrophic events creating turbulence in the environment and introducing dust, smoke and other foreign materials to the atmosphere contributing to the noise. When a packet of size $\mathrm{N}$ bits is transmitted wireless $\mathrm{K}$ bits are correct. If $0 \leq K \leq N$ If $\mathrm{K}<\mathrm{N}$ the remaining $\mathrm{N}-\mathrm{K}$ are not correct. $\mathrm{K}$ is a random variable and its size depends on the noise. When the packet is retransmitted then $\mathrm{L}$ bits are correct . If $0 \leq L \leq N<\mathrm{N}$ then $\mathrm{m}$ out of these correct bits come from the original $\mathrm{K}$ and $\mathrm{L}-\mathrm{m}$ come from the $\mathrm{N}-\mathrm{K}$. If $\mathrm{X}$ is a random variable signifying the number of correct bits in the second transmission then

$$
p(X=K)=\frac{\left(\begin{array}{l}
K \\
m
\end{array}\right)\left(\begin{array}{l}
N-K \\
L-m
\end{array}\right)}{\left(\begin{array}{l}
N \\
L
\end{array}\right)}
$$

Is a hypergeometric distribution. It is easy to prove that all transmissions after the first are hypergeometric distributions. In our strategy we allow the transmitter to keep sending the same packet with the receiver sending an acknowledgement that the packet was received after the receiver verifies that the packet is received correctly, at that time the transmitter sends the next packet. Not sending acknowledgements while a packet is not all correct saves time. In addition our algorithm employs a hidden Markov algorithm to optimize the size of the packet, real time, depending on the environmental noise.

\section{The Intelligent Systems}

The premise of AI is that it needs to have four characteristics. The first characteristic is perception. In our case perception is the ability to understand our environment as its characterized by its environmental, geological, and fire characteristics. So WSN provides the $\mathrm{Co}, \mathrm{Co}_{2}$ concentration in various locations of the forest, they also provide the temperature, the wind speed and wind direction, and humidity. The SODAR provides the wind speed at various altitudes; the LIDAR provides ground slopes, shapes, and accurate geometric dimensions of each part of the forest. It also provides accurately the area of the fire. Our sensors in general provide the "touch and feel" which is the perception in our case. The second characteristic is the learning. The learning in our case is achieved by our algorithms that take as input the sensor data, include the data from the imagers, and learn about their environment. The learning has several stages. One stage is using past data to teach our intelligent system to recognize the environment they represent. So when the system is faced with a new situation to be able to characterize it correctly. The other is to understand transitions representing escalation or de-escalation of the catastrophic event. The third characteristic of $\mathrm{AI}$ is reasoning. In that regard our forest fire system has 10 scales from 0 to 10 . Ten being the most catastrophic, and the AI is programmed so that it can reason it out based on what it has learned and develop a strategy based on the available resources to reduce the fire intensity from $i$, where $i$ is greater than 0 and less than or equal to 10 , to i- 1 in the least amount of time. The fourth component of $\mathrm{AI}$ is natural languages. There is no need for that component in this case. However there is a component similar to that. That component is the ability for the airplane to transmit very accurate computational photography and video real time as well as advice to the firefighters. The computational intensity of our system requires a great deal of computer power. This power is being delivered by a number of stratix-10 Intel-Altera FPGAs [13-14]. The $14 \mathrm{~nm}$ technology is capable of delivering 39.5 teraflops per second per FPGA. It is only with this kind of power that a complex system like this could be possible. Proper weight initialization and training sampling size are very critical to the convergence of the AI system [15-17].

\section{Conclusion}

In this research paper we describe an autonomous intelligent UAS to prevent, detect fires, and provide advice to the firefighters in case of fires to effectively fight the fires. The system includes the solar powered airplane the specifications and design of which is given in this paper. An enhanced wireless communication network system, with the enhancement described in the previous section. The artificial intelligence system includes an AI subsystem providing the autonomy, an AI providing the prevention and 
detection, and an AI system providing real time advice to the firefighters. This is a beginning to end original design satisfying the goals described in this paper, and not an integrated project with engineering compromises.

\section{References}

1. Cappellini V, Mattii L, Mecocci A. An Intelligent System for Automatic Fire Detection in forests. Recent Issues in Pattern Analysis and Recognition. 1989;399:351-364.

2. Millan L, Sanchez G, Nakano M, Toscano-Medina K, Perez-Meana H, Rojas L. An early fire detection algorithm using IP cameras. Sensor. 2012;12:5670-5686. doi:10.3390/s120505670

3. Yuan C, Zhang Y, Liu Z. A Survey on Technologies for Automatic Forest Fire Monitoring, Detection, and Fighting Using UAVs and Remote Sensing Techniques. Can Jour for Res. 2015;45(7):783-792.

4. Qian Y, Yan G, Duan S, Kong X. A contextual fire detection Algorithm for simulated HJ-1B imagery. Sensors (Basel). 2009;9(2):961-979. doi: $10.3390 / \mathrm{s} 90200961$

5. Yoon SH, Min J. An Intelligent Automatic Early Detection System of Foreest Fire Smoke Signatures Using Gausian Mixture Model. Jour Inf Process Syst. 2013;9(4):621-632. doi: 10.3745/JIPS.2013.9.4.621

6. Stula M, Krstinic D, Seric L. Intelligent Forest Fire Monitoring System. Inf Syst Front. 2012;14(3):725-739.

7. Cruz H, Eckert M, Meneses J, Martinez JF. Efficient Forest Fire Detection Index for Application in Unmanned Aerial Systems (UASs). Sensors. 2016;16(6):893. doi: 10.3390/s16060893

8. Molina-Pico A, Cuesta-Frau D, Araujo A, Alejandre J, Rozas A. Forest Monitoring and Wildland Early Fire Detection by a Hierarchical Wireless Sensor Network. Journal of Sensors. 2016;2016.
9. Yuan C, Liu Z, Zhang Y. UAV-based forest fire detection and tracking using image processing techniques. Proceedings of the IEEE International Conference on Unmanned Aircraft Systems (ICUAS 2015). 2016:639-643.

10. Canadian Fire Weather Index. Weather and Life. Available from: https://www.islandnet.com/ see/weather/life/canfireindex.htm

11. Bobbe T. The Role of UAV Technology in Forest Service Natural Resource Management. Workshop on UAV's for Land Management and Coastal Zone Dynamics. 2005.

12. Rathgeb B, Cheng Q. Utilizing the IEE 802.16 Standard for Homeland Security Applications. SPIE Defense and Security Fence and Security Symposium: Technologies for Homeland Security Law Enforcement. 2005.

13. Maditinos Z, Vassiliadis C. Mega fires: Can they be Managed Effectively? Disaster Prevention and Management: An International Journal. 2011;20(1):41-52.

14. Harris SL, Harris DM. Digital Design and Computer Architecture: Arm Edition. Morgan Kaufmann Publishers Inc. 2015.

15. Harris SL, Harris DM. Harris, Digital Design and Computer Architecture. Morgan Kaufmann Publishers Inc. 2012.

16. Yfantis EA. A New Era with New Computer Science Challenges. Keynote Speech The 7th IEEE Annual Computing and Communication Conference. 2017.

17.Zamora E, Nakakuni M, Yfantis EA. Quantitative Measures to evaluate Neural Network Weight Initialization Strategies. The 7th IEEE Annual Computing and Communication Conference. 2017:31-37.

18. Yfantis EA, Nakakuni M, Zamora E. Low Bandwidth Transmission Algorithm for Reliable Wireless Communication, The $7^{\text {th }}$ IEEE Annual Computing and Communication Conference. 2017:38-42. 